\title{
BMJ Open Diagnostic features, management and prognosis of type 2 myocardial infarction compared to type 1 myocardial infarction: a systematic review and meta-analysis
}

\author{
Kyle White (D) , ${ }^{1,2}$ Mansey Kinarivala, ${ }^{1}$ Ian Scott (D) ${ }^{1,2}$
}

To cite: White K, Kinarivala M, Scott I. Diagnostic features, management and prognosis of type 2 myocardial infarction compared to type 1 myocardial infarction: a systematic review and meta-analysis. BMJ Open 2022:12:e55755. doi:10.1136/ bmjopen-2021-055755

- Prepublication history and additional supplemental material for this paper are available online. To view these files, please visit the journal online (http://dx.doi.org/10.1136/ bmjopen-2021-055755).

Received 23 July 2021 Accepted 28 January 2022

Check for updates

(C) Author(s) (or their employer(s)) 2022. Re-use permitted under CC BY-NC. No commercial re-use. See rights and permissions. Published by BMJ.

${ }^{1}$ Internal Medicine and Clinical Epidemiology, Princess Alexandra Hospital, Woolloongabba, Queensland, Australia

${ }^{2}$ School of Clinical Medicine, University of Queensland, Brisbane, Queensland, Australia

Correspondence to

Dr Kyle White;

kyle.white@health.qld.gov.au

\section{ABSTRACT}

Importance Distinguishing type 2 (T2MI) from type 1 myocardial infarction (T1MI) in clinical practice can be difficult, and the management and prognosis for T2MI remain uncertain.

Objective To compare precipitating factors, risk factors, investigations, management and outcomes for T2MI and T1Ml.

Data sources Medline and Embase databases as well as reference list of recent articles were searched January 2009 to December 2020 for term 'type 2 myocardial infarction'.

Study selection Studies were included if they used a universal definition of $\mathrm{Ml}$ and reported quantitative data on at least one variable of interest.

Data extraction and synthesis Data were pooled using random-effect meta-analysis. Risk of bias was assessed using Newcastle-0ttawa quality assessment tool. Preferred Reporting Items for Systematic Reviews and Metaanalyses guidelines were followed. All review stages were conducted by two reviewers.

Main outcomes and measures Risk factors, presenting symptoms, cardiac investigations such as troponin and angiogram, management and outcomes such as mortality.

Results 40 cohort studies comprising 98930 patients with $\mathrm{T} 1 \mathrm{MI}$ and 13803 patients with T2MI were included. Compared with $\mathrm{T} 1 \mathrm{MI}$, patients with $\mathrm{T} 2 \mathrm{MI}$ were: more likely to have pre-existing chronic kidney disease (OR 1.87; $95 \%$ $\mathrm{Cl} 1.53$ to 2.28) and chronic heart failure (OR 2.35; 95\% Cl 1.82 to 3.03), less likely to present with typical cardiac symptoms of chest pain (OR $0.19 ; 95 \% \mathrm{Cl} 0.13$ to 0.26 ) and more likely to present with dyspnoea (OR 2.64; $95 \%$ $\mathrm{Cl} 1.86$ to 3.74$)$; more likely to demonstrate non-specific ST-T wave changes on ECG (OR 2.62; $95 \% \mathrm{Cl} 1.81$ to 3.79 ) and less likely to show ST elevation (OR 0.22; $95 \% \mathrm{Cl} 0.17$ to 0.28 ); less likely to undergo coronary angiography ( $\mathrm{OR}$ $0.09 ; 95 \% \mathrm{Cl} 0.06$ to 0.12 ) and percutaneous coronary intervention (OR $0.06 ; 95 \% \mathrm{Cl} 0.04$ to 0.10 ) or receive cardioprotective medications, such as statins (OR 0.25 ; $95 \% \mathrm{Cl} 0.16$ to 0.38 ) and beta-blockers (OR 0.45; 95\% $\mathrm{Cl} 0.33$ to 0.63 ). T2Ml had greater risk of all cause 1 year mortality (OR $3.11 ; 95 \% \mathrm{Cl} 1.91$ to 5.08 ), with no differences in short-term mortality (OR 1.34; 95\% Cl 0.63 to 2.85 )
Strengths and limitations of this study

Inclusion of all contemporary cohort studies in the troponin era.

- Analyses of large population of patients with type 2 myocardial infarction and type 1 myocardial infarction which provided high level of precision.

- Wide array of clinically significant variables assessed providing a comprehensive analysis.

- Analysis of crude mortality only was possible due to lack of individual patient data.

Conclusion and relevance This review has identified clinical, management and survival differences between $\mathrm{T} 2 \mathrm{MI}$ and $\mathrm{T} 1 \mathrm{Ml}$ with greater precision and scope than previously reported. Differential use of coronary revascularisation and cardioprotective medications highlight ongoing uncertainty of their utility in T2Ml compared with $\mathrm{T} 1 \mathrm{MI}$.

\section{INTRODUCTION}

The clinical definition of myocardial infarction (MI) has evolved over time. The 2007 Universal Definition of Myocardial Infarction included a subset of MI that was secondary to aetiologies unrelated to underlying occlusive coronary artery disease. ${ }^{1}$ In 2012, the Third Universal Definition of Myocardial Infarction Consensus Document ${ }^{2}$ gave rise to the aetiological distinction between T1MI, defined as MI due to plaque erosion and/or rupture, and T2MI, defined as MI caused by increased oxygen demand or decreased blood supply, in the absence of acute plaque rupture or coronary thrombosis. More recently, in 2018, the Fourth Universal definition of MI updated concepts of T2MI regarding specific situations associated with oxygen demand and supply imbalance and the relevance of the presence or 
absence of underlying coronary artery disease to therapy and prognosis ${ }^{3}$ (see online supplemental table S1 for more detail).

In clinical practice, distinguishing T2MI from T1MI based on clinical presentation, ECG features and cardiac troponin ( $\mathrm{cTn}$ ) values can be difficult. In the absence of randomised controlled trials that have evaluated different investigational and therapeutic interventions in patients with T2MI, uncertainty remains around the appropriate management of such patients, particularly those with known or suspected coronary artery disease. Past reviews have assessed one or more attributes of T2MI in comparison to $\mathrm{T} 1 \mathrm{MI}^{4-8}$ but, to our knowledge, none have undertaken a comprehensive analysis of symptoms, physical signs, investigation results, management regimens and clinical outcomes, both short and long term, of T2MI versus T1MI.

We undertook a systematic review of observational studies with the aims of identifying diagnostic and investigational findings which can assist clinicians to better distinguish T2MI from T1MI, and compare T2MI with T1MI in defining differences in management strategies and clinical outcomes.

\section{METHODS}

\section{Study design}

The review was undertaken in accordance with recommendations of the Cochrane Collaboration and Preferred Reporting Items for Systematic Reviews and MetaAnalyses (PRISMA) statement. ${ }^{9}$ Our review was registered on PROSPERO prior to commencement (registration number: CRD42021237746). Medline and Embase databases were searched for all studies published between 1 January 2009, and 31 December 2020, using search terms to identify all studies related to T2MI (see online supplemental table S2). Reference lists of all relevant articles were also assessed to identify additional relevant studies. The study PRISMA flow chart is shown in online supplemental figure S1. January 2009 was chosen as the start date for the literature search in order to restrict our analyses to contemporary studies in the troponin era that employed formal definitions of T2MI which were only devised from 2007 onwards.

Studies were included if they: (1) compared patient populations with T2MI and T1MI, (2) used a universal definition of MI, (3) included at least one variable of interest, (4) were available as full text in English and (5) were either a randomised control trial or comparative observational study. Studies were excluded if: (1) no full text was available, (2) duplicate data were used or (3) less than 200 participants in total were included. Initial screening of titles and abstracts for eligible studies was performed independently by two authors (MK, KW), as was full-text review for inclusion, with any differences in review settled by consensus agreement.

\section{Data collection and synthesis}

Data pertaining to all variables of interest were collected from all included studies using a standardised proforma by one author (MK) and independently reviewed by the second author (KW). These variables comprised: study dates, design, sample size, definition used to define T2MI and T1MI, patient demographics, pre-existing medical conditions, precipitating factors, clinical symptoms, ECG findings, laboratory values, echocardiographic results, any clinical interventions or medical treatments administered, and clinical outcomes observed.

Data on variables reported as, or able to be converted to, raw numbers, were pooled from all studies and subject to comparative meta-analysis using Review Manager (RevMan, Computer program. Version 5.3. Copenhagen: The Nordic Cochrane Centre, The Cochrane Collaboration, 2014). For each variable, the odds ratio (OR) comparing T2MI to T1MI, and its 95\% confidence interval (CI), was calculated and weighted using the random effects method. As specified in the registered study protocol, the random effects method was used in anticipation of study heterogeneity of at least moderate degree $\left(\mathrm{I}^{2}\right.$ statistic of heterogeneity $\left.>50 \%\right) .{ }^{10}$ In addition to the weighted OR, we also report the crude total event rates for each variable subject to meta-analysis in order to provide a more clinically meaningful estimate of the prevalence of these events in each patient group in view of the large sample sizes. Studies reporting mean or median values only were reproduced as reported in the original study.

Risk of bias within each study was assessed using the Newcastle-Ottawa quality assessment tool for cohort studies, ${ }^{11}{ }^{12}$ with scores 7-8 denoting good quality studies, 4-6 fair quality, and 0-3 poor quality. Publication bias was assessed using funnel plots.

\section{Patient and public involvement}

We did not seek patient or public comment in designing the study.

\section{RESULTS}

A total of 40 studies were included for analysis ${ }^{13-52}$ and their characteristics are summarised in online supplemental table S3. They comprised a total of 127620 participants of whom 98930 participants $(77.5 \%)$ were classified as T1MI and $13803(10.8 \%)$ as T2MI. In the following text, we report key findings; more information and forest plots for each analysis involving more than one study and more than 100 total cases can be found in the online supplement, online supplemental figure S2-S44.

The 2007 definition $^{1}$ was used in $7(17.5 \%)$ studies, ${ }_{15} 162729434452$ the 2012 definition $^{2}$ in $25(62.5 \%)$ studies, ${ }^{13} 17$ 19-21 23-26 30-35 $3739404245-485051$ and the 2018 definition $^{3}$ in 8 (20\%) studies. $^{14} 18222836384149$ Of the 40 studies, $17(42.5 \%)$ were prospective $e^{15} 161819$ $22293334363743-4547485051$ and $23(57.5 \%)$ were retrospective. ${ }^{13} 14172021$ 23-28 30-32 $3538-42464952$ 
Table 1 Pre-existing medical conditions in patients with type 2 myocardial infarction (T2MI) versus type 1 myocardial infarction (T1MI)

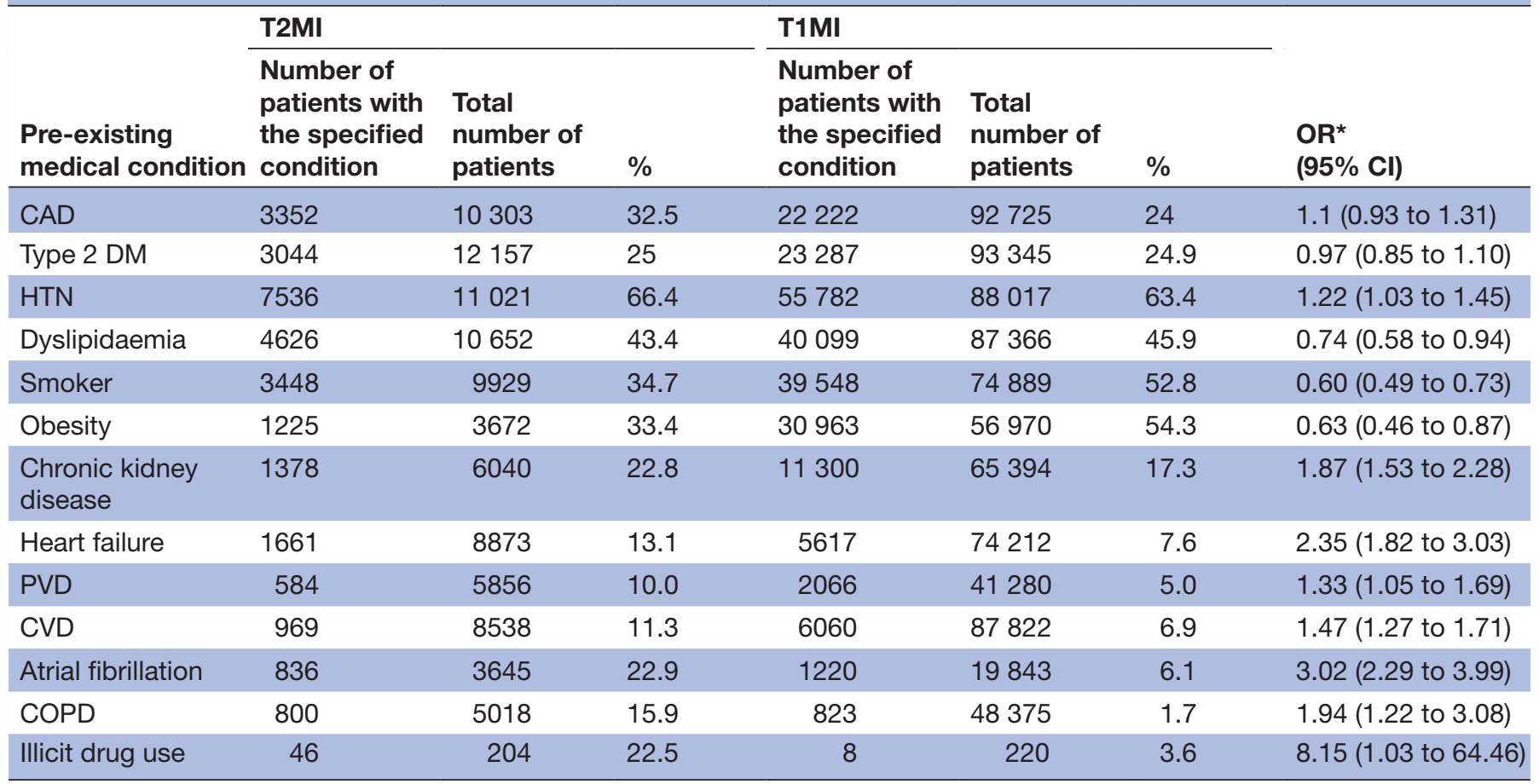

${ }^{*}$ Comparing patients with T2MI with those with $\mathrm{T} 1 \mathrm{Ml}$, with OR adjusted according to study weighting using random effects meta-analysis. $\mathrm{BMI}$, body mass index; CAD, coronary heart disease; COPD, chronic obstructive pulmonary disease; CVD, cerebrovascular disease; DM, diabetes mellitus; HTN, hypertension; PVD, peripheral vascular disease.

\section{Risk-of-bias assessment}

Of the 40 studies, $31(77.5 \%)$ were assessed as good quality, ${ }^{13} 15-192223$ 27-35 37-46 48 50-52 6 (15\%) as fair quality, ${ }^{14}$ $2124-2649$ and $3(7.5 \%)$ as poor quality, ${ }^{203647}$ as summarised in online supplemental table $\mathrm{S} 4$. Selection bias resulting in unrepresentative cohorts such as admission criteria to coronary care units or entry criteria into MI registries favouring T1MI, ${ }^{142024-26364749}$ absence of independent adjudication of MI type as T1MI or T2MI, ${ }^{36} 3847$ noncomparability of T1MI and T2MI cohorts, ${ }^{20} 242547$ poorly specified outcome measures ${ }^{36} 3847$ and short follow-up period resulting in few events ${ }^{14202436}$ comprised most forms of bias.

Funnel plots for in-hospital and 1-year all-cause mortality showed no asymmetry (online supplemental figures S45 and S46). Funnel plots for all other analyses showed similar results (available on request).

\section{Participant characteristics}

Patients with T1MI had a median age range of 60-82 years in the included studies that did not select a specific age population, compared with a median age range of 62-81 years in patients with T2MI. The sex distribution was also similar, with $58.4 \%$ and $53 \%$ of patients with T1MI and T2MI being male, respectively.

Regarding pre-existing medical conditions (table 1), patients with T2MI compared with patients with T1MI were more likely to have chronic kidney disease $(22.8 \%$ vs $17.3 \%$; OR 1.87; $95 \%$ CI 1.53 to 2.28 ), chronic heart failure (13.1\% vs 7.6\%; OR 2.35; 95\% CI 1.82 to 3.03 ), atrial fibrillation (22.9\% vs $6.1 \%$; OR $3.02 ; 95 \%$ CI 2.29 to 3.99 ) and hypertension (66.4\% vs $63.4 \%$; OR 1.22 ; $95 \%$ CI 1.03 to 1.45$)$. Patients with T2MI were less likely to have dyslipidaemia (43.4\% vs $45.9 \%$; OR $0.74 ; 95 \%$ CI 0.58 to 0.94 ) and smoking history ( $34.7 \%$ vs $52.8 \%$; OR $0.6 ; 95 \%$ CI 0.49 to 0.73$)$. There was no difference in the prevalence of type 2 diabetes mellitus or ischaemic heart disease between the two groups.

\section{Precipitating factors}

Less than half of the studies $(n=18 ; 45 \%)$ included data on precipitating factors associated with T2MI. ${ }^{1315171921-242731323540444550-52}$ Data on each precipitating factor were not consistently available across the studies; for example, only 17 studies representing $45 \%$ of patients with T2MI assessed the presence of arrythmia.

The most common precipitants were sepsis $(35.9 \%)$ and heart failure $(35.9 \%$, followed by arrythmia $(29.8 \%)$ (online supplemental table S5), with non-cardiac surgery being deemed a cause in $12.2 \%$ of cases where data for this variable were collected.

\section{Presenting clinical features}

As summarised in online supplemental table S6, compared with patients with T1MI, patients with T2MI were less likely to present with typical cardiac symptoms of chest pain $(58.6 \%$ vs $88.4 \%$; OR $0.19 ; 95 \%$ CI 0.13 to $0.26)$ or discomfort in the arm or shoulder $(8.5 \%$ vs $35 \%$; 
OR 0.18 ; $95 \%$ CI 0.11 to 0.3 ), but more likely to present with dyspnoea (27.1\% vs $10.6 \%$; OR $2.64 ; 95 \%$ CI 1.86 to 3.74).

\section{Investigations}

ECG findings on presentation (online supplemental table S7) such as ST elevation ( $14.1 \%$ vs $44.2 \%$; OR 0.22 ; $95 \%$ CI 0.17 to 0.28$)$ and pathological $Q$ waves $(6.7 \%$ vs 20.8\%; OR 0.38 ; $95 \%$ CI 0.20 to 0.71 ) were less evident in T2MI than in T1MI. In contrast, non-specific ST-T wave changes $(24.7 \%$ vs $10.8 \%$; OR 2.62; $95 \%$ CI 1.81 to 3.79$)$, and atrial arrythmias (21\% vs $6.6 \%$; OR $4.99 ; 95 \%$ CI 3.14 to 7.93) were more common among T2MI. No differences between groups were seen in the frequency of ST depression or $\mathrm{T}$ wave inversion.

Among the 40 studies, 4 studies (10\%) reported the use of high-sensitivity cTn assays, 21 (53\%) reported sensitive assays and $14(35 \%)$ did not specify what generation assay was used (online supplemental tables S3a and S3b). The results of troponin assays were reported in $26(65 \%)$ studies, specific to cTnI assays in 19 studies, cTnT in 5, both assays in 1, while another did not specify the assay used. Only two of these studies reporting troponin failed to state the upper limit of normal (ULN) of the assay used. $^{23} 31$ The troponin assays, and therefore units and reference ranges, varied between the studies, preventing direct comparison of troponin values. As a result, we converted troponin values to a multiple of the ULN for each assay to allow direct comparison (online supplemental table S8). For peak troponin, patients with T1MI had a higher and wider range of between 5 and 1702 times the ULN compared with patients with T2MI with a range of 2.8-447 times the ULN. Studies yielded mixed results as to whether the magnitude of change (or delta) in serial cTn assays was more predictive of T2MI or T1MI compared with absolute values of peak levels. ${ }^{33}$ Lowering the diagnostic threshold for troponin with the advent of more sensitive assays has increased the numbers of patients identified with T2MI by up to $50 \%,{ }^{36}$ with more recent studies showing the incidence of T2MI equalling or exceeding that of T1MI. ${ }^{15} 3336$

Echocardiography was less frequently performed among patients with T2MI than those with T1MI $(47.9 \%$ vs $55.5 \%$; OR 0.44; $95 \%$ CI 0.20 to 0.96 ) and when reported (online supplemental table S7), there was no difference in the prevalence of regional wall motion abnormalities or the level of left ventricular (LV) function, with reported median LV ejection fraction being $42.3 \%-55 \%$ in patients with T1MI and 40\%-56\% in patients with T2MI.

Coronary angiography was also less frequently performed among patients with T2MI than in those with T1MI ( $34.1 \%$ vs $85.5 \%$; OR 0.09 ; $95 \%$ CI 0.06 to 0.12 , online supplemental table $\mathrm{S} 7$ ). When performed, patients with T2MI were less likely to demonstrate obstructive coronary artery disease (34\% vs $44.9 \%$; OR 0.16 ; $95 \%$ CI 0.05 to 0.54 ), with obstruction variously defined as $50 \%-70 \%$ occlusion of one or more vessels.

\section{Management}

Patients with T2MI, compared with patients with T1MI, were significantly less likely to receive conventional cardioprotective medications (table 2), comprising betablockers ( $58.3 \%$ vs $76.3 \%$; OR 0.45 ; $95 \%$ CI 0.33 to 0.63 ), antiplatelet agents $(70.8 \%$ vs $88.5 \%$; OR $0.25 ; 95 \%$ CI 0.16 to 0.38 ) and statins ( $52.9 \%$ vs $87.6 \%$; OR 0.25 ; $95 \%$ CI 0.16 to 0.38 ). Of note, patients with T2MI were more likely to receive diuretics $(44.8 \%$ vs $13.6 \%$; OR 1.98 ; $95 \%$

Table 2 Pharmacological management and invasive interventions in patients with type 2 myocardial infarction (T2MI) versus type 1 myocardial infarction (T1MI)

\begin{tabular}{|c|c|c|c|c|c|c|c|}
\hline \multirow[b]{2}{*}{ Intervention } & \multicolumn{3}{|l|}{ T2MI } & \multicolumn{3}{|l|}{ T1MI } & \multirow[b]{2}{*}{$\begin{array}{l}\text { OR* } \\
(95 \% \mathrm{CI})\end{array}$} \\
\hline & $\begin{array}{l}\text { No. patients } \\
\text { receiving } \\
\text { intervention }\end{array}$ & $\begin{array}{l}\text { Total } \\
\text { number of } \\
\text { patients }\end{array}$ & $\%$ & $\begin{array}{l}\text { No. patients } \\
\text { receiving } \\
\text { intervention }\end{array}$ & $\begin{array}{l}\text { Total } \\
\text { number of } \\
\text { patients }\end{array}$ & $\%$ & \\
\hline \multicolumn{8}{|l|}{ Medication } \\
\hline Beta blockers & 4967 & 8523 & 58.3 & 63431 & 83157 & 76.3 & 0.45 (0.33 to 0.63$)$ \\
\hline ACEI/ARB & 3766 & 7842 & 48 & 56253 & 81793 & 68.8 & 0.52 (0.40 to 0.67$)$ \\
\hline Anticoagulants & 1519 & 5255 & 28.9 & 15754 & 62415 & 25.2 & $1.87(1.06$ to 3.30$)$ \\
\hline Antianginal agents & 1281 & 2191 & 58.5 & 38955 & 42768 & 91.1 & $0.61(0.21$ to 1.74$)$ \\
\hline Diuretics & 1336 & 2985 & 44.8 & 6211 & 45779 & 13.6 & 1.98 (1.37 to 2.86$)$ \\
\hline Statins & 3418 & 6455 & 52.9 & 56875 & 64942 & 87.6 & 0.25 (0.16 to 0.38$)$ \\
\hline
\end{tabular}

Comparing patients with T2MI with those with T1MI, with OR adjusted according to study weighting using random effects meta-analysis. ACEI, ACE inhibitors; ARB, angiotensin receptor blockers; CABG, coronary artery bypass graft; PCl, percutaneous coronary intervention. 
Table 3 Outcomes in patients with type 2 myocardial infarction (T2MI) versus type 1 myocardial infarction (T1MI)

\begin{tabular}{|c|c|c|c|c|c|c|c|}
\hline \multirow[b]{2}{*}{ Outcomes } & \multicolumn{3}{|l|}{ T2MI } & \multicolumn{3}{|l|}{ T1MI } & \multirow[b]{2}{*}{$\begin{array}{l}\text { OR* }^{\star} \\
(95 \% \mathrm{Cl})\end{array}$} \\
\hline & $\begin{array}{l}\text { No. patients } \\
\text { with } \\
\text { outcome }\end{array}$ & $\begin{array}{l}\text { Total } \\
\text { number of } \\
\text { patients }\end{array}$ & $\%$ & $\begin{array}{l}\text { No. patients } \\
\text { with } \\
\text { outcome }\end{array}$ & $\begin{array}{l}\text { Total } \\
\text { number of } \\
\text { patients }\end{array}$ & $\%$ & \\
\hline CV in-hospital mortality & 184 & 2109 & 8.7 & 331 & 6248 & 5.3 & 1.61 (1.17 to 2.22$)$ \\
\hline All-cause in-hospital mortality & 667 & 5321 & 12.5 & 1508 & 25997 & 5.8 & 1.94 (1.35 to 2.79$)$ \\
\hline Short-term all-cause mortality & 204 & 887 & 23.0 & 250 & 1998 & 12.5 & 1.34 (0.63 to 2.85$)$ \\
\hline 2-year all-cause mortality & 246 & 926 & 26.6 & 428 & 2587 & 16.5 & $1.63(1.11$ to 2.41$)$ \\
\hline 3-year all-cause mortality & 193 & 525 & 36.8 & 710 & 4305 & 16.5 & $2.00(1.07$ to 3.76$)$ \\
\hline Long-term all-cause mortality & 1453 & 2708 & 53.7 & 1320 & 4633 & 28.5 & $3.24(2.73$ to 3.84$)$ \\
\hline
\end{tabular}

${ }^{*}$ Comparing patients with T1MI with those with T2MI, with OR adjusted according to study weighting using random effects meta-analysis. $\mathrm{CV}$, cardiovascular.

CI 1.37 to 2.86$)$ or anticoagulants $(28.9 \%$ vs $25.2 \%$; OR $1.87 ; 95 \%$ CI 1.06 to 3.30$)$.

Percutaneous coronary intervention (PCI) $(21.1 \%$ vs $78.0 \%$; OR 0.06 ; $95 \%$ CI 0.04 to 0.10 ) and coronary artery bypass surgery $(2.9 \%$ vs $6.4 \%$; OR $0.23 ; 95 \%$ CI 0.12 to 0.45 ) were also significantly less likely to be performed in patients with T2MI than patients with T1MI.

\section{Prognosis}

Patients with T2MI had significantly increased risk of allcause death compared with patients with T1MI in both short-term and long-term follow-up (table 3). Specifically, compared with patients with T1MI, T2MI demonstrated increased all-cause mortality in-hospital $(12.5 \%$ vs $5.8 \%$; OR 1.94 ; $95 \%$ CI 1.35 to 2.79 , online supplemental figure S40), at 1 year ( $18.9 \%$ vs $5.4 \%$; OR 3.11 ; $95 \%$ CI 1.91 to 5.08 , figure 1$)$ and at $5-10$ years $(53.7 \%$ vs $28.5 \%$, OR 3.24; $95 \%$ CI 2.73 to 3.84 , figure 2 ). In contrast, there were no differences between patients with T2MI and T1MI in the risk of short-term mortality at $120-180$ days $(23.0 \%$ vs $12.5 \%$; OR 1.34 ; $95 \%$ CI 0.63 to 2.85$)$.

\section{DISCUSSION}

To our knowledge, this is the most comprehensive systematic review and meta-analysis of contemporary studies comparing T2MI with T1MI in the troponin era, comprising 127620 patients from 40 cohort studies across 14 countries, and which used formal definitions of T2MI and T1MI. Up to three quarters of all MIs in routine care can be T2MI, ${ }^{33}{ }^{34}$ and distinguishing T2MI from T1MI on clinical criteria is often challenging. The management strategies used by clinicians in real-world practice for T2MI often vary, and the clinical outcomes of T2MI compared with T1MI, particularly over the long term, have been uncertain. This review provides information that helps characterise these two groups of patients according to multiple variables and which may assist in clinical decision-making and prognostication.

In this review, patients with T2MI demonstrated more medical comorbidities than patients with T1MI, as noted in a recent meta-analysis. ${ }^{6}$ Our review highlighted the much higher incidence of pre-existing generalised vascular disease, atrial fibrillation, renal impairment and heart failure among patients with T2MI.

\begin{tabular}{|c|c|c|c|c|c|c|c|c|}
\hline \multirow[b]{2}{*}{ Study or Subgroup } & \multicolumn{2}{|c|}{ T2MI } & \multicolumn{2}{|c|}{ T1MI } & \multirow[b]{2}{*}{ Weight } & \multirow{2}{*}{$\begin{array}{c}\text { Odds Ratio } \\
\text { M-H, Random, } 95 \% \mathrm{Cl}\end{array}$} & \multirow{2}{*}{\multicolumn{2}{|c|}{$\begin{array}{c}\text { Odds Ratio } \\
\mathrm{M}-\mathrm{H}, \text { Random, } 95 \% \mathrm{Cl}\end{array}$}} \\
\hline & Events & Total & Events & Total & & & & \\
\hline Arora 2018 & 89 & 264 & 96 & 775 & $13.1 \%$ & $3.60[2.58,5.02]$ & & - \\
\hline Chapman 2020 & 258 & 1121 & 720 & 4981 & $13.7 \%$ & $1.77[1.51,2.08]$ & & $=$ \\
\hline El haddad 2012 & 84 & 295 & 28 & 512 & $12.4 \%$ & $6.88[4.36,10.87]$ & & - \\
\hline Furie 2019 & 80 & 206 & 93 & 349 & $12.9 \%$ & $1.75[1.21,2.52]$ & & $=$ \\
\hline Lopez Cuenca 2016 & 27 & 117 & 102 & 707 & $12.3 \%$ & $1.78[1.10,2.87]$ & & 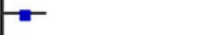 \\
\hline Radovanovic 2017 & 14 & 1091 & 117 & 13828 & $11.8 \%$ & $1.52[0.87,2.66]$ & & $t=-$ \\
\hline Saaby 2014 & 65 & 119 & 25 & 360 & $11.9 \%$ & $16.13[9.37,27.77]$ & & - \\
\hline Stein 2014 & 15 & 127 & 118 & 2691 & $11.7 \%$ & $2.92[1.65,5.16]$ & & 7 \\
\hline Total $(95 \% \mathrm{Cl})$ & & 3340 & & 24203 & $100.0 \%$ & $3.11[1.91,5.08]$ & & \\
\hline Total events & 632 & & 1299 & & & & & \\
\hline $\begin{array}{l}\text { Heterogeneity: Tau² } \\
\text { Test for overall effect }\end{array}$ & $\begin{array}{l}0.45 ; \text { Chi }^{2} \\
Z=4.55(\end{array}$ & $\begin{array}{l}=94.64 \\
P<0.00\end{array}$ & $\begin{array}{l}4, d f=7( \\
0001)\end{array}$ & $P<0.00$ & $0001) ; 1^{2}$ & $93 \%$ & $\begin{array}{l}0.01 \quad 0.1 \\
\text { Favours T1Ml }\end{array}$ & $\begin{array}{c}10 \\
\text { Favours T2Ml }\end{array}$ \\
\hline
\end{tabular}

Figure 1 Forest plot of 1-year all-cause mortality of patients with type 2 myocardial infarction (T2MI) compared with patients with type 1 myocardial infarction (T1MI). 


\begin{tabular}{|c|c|c|c|c|c|c|c|c|}
\hline Study or Subgroup & \multicolumn{2}{|c|}{ T2MI } & T1MI & Total & Weight & $\begin{array}{c}\text { Odds Ratio } \\
\text { M-H, Random, } 95 \% \mathrm{Cl}\end{array}$ & \multicolumn{2}{|c|}{$\begin{array}{c}\text { Odds Ratio } \\
\text { M- } \mathrm{H}, \text { Random, } 95 \% \mathrm{Cl}\end{array}$} \\
\hline Chapman 2018 & 268 & 429 & 430 & 1171 & $28.3 \%$ & $2.87[2.28,3.61]$ & & $=$ \\
\hline Raphael 2020 & 766 & 1054 & 638 & 1365 & $36.2 \%$ & $3.03[2.55,3.60]$ & & $=$ \\
\hline Singh 2020 & 419 & 1225 & 252 & 2097 & $35.5 \%$ & $3.81[3.19,4.54]$ & & $=$ \\
\hline Total $(95 \% \mathrm{Cl})$ & & 2708 & & 4633 & $100.0 \%$ & $3.24[2.73,3.84]$ & & 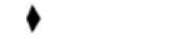 \\
\hline Total events & 1453 & & 1320 & & & & & \\
\hline $\begin{array}{l}\text { Heterogeneity: Tau }= \\
\text { Test for overall effect }\end{array}$ & $\begin{array}{l}0.01 ; \mathrm{Ch} F \\
\mathrm{Z}=13.42\end{array}$ & $\begin{array}{l}=4.84 \\
(P<0 .\end{array}$ & $\begin{array}{l}d f=2(P \\
00001)\end{array}$ & $=0.09$ & ); $\left.\right|^{2}=59 \%$ & & $\begin{array}{ccc}0.01 & 0.1 & 1 \\
\text { FavoursT1MI }\end{array}$ & $\begin{array}{c}10 \\
\text { Favours T2MI }\end{array}$ \\
\hline
\end{tabular}

Figure 2 Forest plot of long-term all-cause mortality of patients with type 2 myocardial infarction (T2MI) compared with patients with type 1 myocardial infarction (T1MI).

Sepsis $^{10} 1627$ and anaemia ${ }^{51}$ ranked highly as triggers, together with other acute cardiac events such as valve dysfunction or arrhythmias. In one study, a more favourable prognosis in T2MI was seen when the principal trigger was arrhythmia compared with non-cardiac surgery, hypotension, anaemia or hypoxia. ${ }^{29}$ In another study, shock syndromes were triggers portending a worse prognosis compared with all other triggers. ${ }^{32}$ In our analysis, non-cardiac surgery as a trigger was less frequent than reported by other investigators ${ }^{26}$ whereby perioperative stressors including blood loss, anaesthesia-induced hypotension and wound infections cause imbalance in myocardial contractility, oxygen demand and blood flow. ${ }^{53}$

Analysis of cTn levels showed uniformly higher values in T1MI than T2MI which accord with one review ${ }^{5}$ reporting cTn values 30\%-94\% higher in patients with T1MI, and which other investigators regard as being highly specific diagnostic markers for T1MI. ${ }^{53}$

Coronary angiography and revascularisation were both performed much less frequently in patients with T2MI than those with T1MI. Treating physicians may perceive invasive strategies as being contraindicated or potentially harmful in the presence of various comorbidities more commonly seen in T2MI and associated with competing mortality risk. In our pooled data, only one in three patients with T2MI who underwent angiography demonstrated obstructive coronary artery disease, although this figure may be an underestimate due to selection bias whereby younger, less multimorbid patients preferentially underwent angiography. In the Catheter Sampled Blood Archive in Cardiovascular Disease (CASABLANCA) cohort study, which enrolled patients with high likelihood of coronary or peripheral artery disease and subjected them to peripheral or coronary angiography, of all those who subsequently suffered incident T2MI, almost half ( $47.7 \%)$ demonstrated $\geq 70 \%$ stenosis in at least 2 major coronary arteries. ${ }^{54}$ These conflicting findings question whether patients presenting with T2MI would benefit from routine use of invasive strategies that define coronary anatomy and, if plaque rupture or critical stenoses are seen, lead to prompt revascularisation, with resultant improvement in patient outcomes. In one study, ${ }^{18}$ angiography unmasked acute plaque rupture in $29 \%$ of patients classified as T2MI. In another study, among 27 of 236 patients with T2MI who underwent revascularisation, the odds of all-cause death were reduced by $67 \%$ compared with the remaining 209 non-revascularised patients. ${ }^{23}$ In contrast, in a third more rigorous study comparing patients with T2MI versus patients with T1MI who received or did not receive PCI within 24 hours of symptom onset, after adjusting results using multivariate logistic regression analysis and inverted probability weighting, ${ }^{15}$ in-hospital mortality was lower in those with T1MI receiving PCI (OR 0.47; $95 \%$ CI 0.40 to 0.55 ; $\mathrm{p}<0.001$ ), but not in those with T2MI receiving PCI (OR 1.09; 95\% CI 0.62 to 1.94; $\mathrm{p}=0.763$ ). However, all these studies are observational, so completion of randomised trials, such as the appropriateness of coronary investigation in myocardial injury and T2MI (ACT-2) trial, which is currently in recruitment, ${ }^{55}$ will hopefully provide a more definitive answer.

Given that a third of patients with T2MI had pre-existing coronary artery disease and most of the remainder had one or more cardiovascular risk factors, the relative underuse of cardioprotective medications is perplexing. It may reflect either clinician uncertainty around their cardioprotective utility in T2MI, or concerns about the potential for adverse interactions with other drugs or diseases commonly seen in multimorbid patients with T2MI. The higher use of diuretics in the T2MI population likely reflects the higher prevalence of heart failure and hypertension. Recognising the heterogeneous mechanisms or conditions leading to T2MI, a phenotype specific approach to the design of future trials will be useful in identifying effective therapies.

An important finding is the much higher all-cause in-hospital and 1-year mortality in patients with T2MI compared with patients with T1MI, similar to the twofold greater mortality rate in T2MI noted in a recent systematic review of nine studies. ${ }^{8}$ In our review, this excess mortality was not driven by an excess of cardiovascular deaths, and likely reflects the competing risks of multiple comorbidities, rather than underlying obstructive coronary artery disease which was seen in $30 \%-50 \%$ of patients with T2MI. ${ }^{26}{ }^{31}$ Studies yielded mixed results as to whether coronary artery disease is an independent predictor of T2MI, ${ }^{20}{ }^{42}$ while others question the angiographic distinction between T2MI and T1MI. For example, in a study of 450 consecutive patients with MI who all underwent coronary angiography within 24 hours of symptom onset, $145(32.2 \%)$ patients had 'true' T1MI 
(acute atherothrombosis and no systemic triggers), 114 (25.3\%) had 'true' T2MI (no atherothrombosis and systemic triggers), $61(13.6 \%)$ patients had neither, and $130(28.9 \%)$ patients had both. ${ }^{41}$ This yielded a discordance of angiographic and clinical definitions of MI type in $42.5 \%$ of patients.

Our review has several limitations. First, in the absence of individual patient data from all included studies, we could not perform multivariate regression analysis in identifying independent predictors of diagnosis, management, or prognosis of T2MI. Second, we did not perform separate analyses of studies according to each version of the universal definition of MI or to different troponin thresholds to define MI, which may impact management and prognosis. However, potential misclassification bias was addressed in a recent study which showed little change in MI classification as type 1 or 2 in the same cohort of emergency admissions to whom the third and fourth universal definitions were applied. ${ }^{56}$ In another study which compared separate T2MI cohorts, as defined by the 2007 and the 2012 definitions, comorbidities and use of cardioprotective medications were less frequent in the 2012 cohort, likely due to less severe MIs being included as a result of the use of more sensitive troponin assays. ${ }^{22}$ Third, we did not collect haemodynamic variables or other physiological measures such as haemoglobin levels and glomerular filtration rate in analysing clinical presentations as these were very inconsistently reported. Fourth, our mortality meta-analyses relied on crude mortality rates reported in each study, with $57 \%$ of studies ${ }^{15-19} 22-28303134353740-42454652$ also undertaking multivariate regression and/or competing risk analyses and reporting adjusted mortality rates. For the T2MI cohorts in general, these rates tended to be lower and the differences in rates compared with those of T1MI were of smaller magnitude. Similarly, we did not attempt subanalyses based on risk stratification using validated risk scores or seek to identify predictive models for mortality, as such analyses were reported in only two studies. ${ }^{26}{ }^{40}$ Fifth, we did not analyse 30-day readmission rates as these were reported in only three studies. ${ }^{13} 1423$ Sixth, we did not perform sensitivity analyses comparing results of prospective vs retrospective studies, as neither group demonstrated less or more risk of bias than the other, or compared results of good quality studies against fair/poor quality studies as the latter comprised only $17 \%$ of all patients. Seventh, as we searched only two databases and did not include grey literature, relevant studies may have been missed. However, in a recent analysis, searching Medline and Embase combined yielded 93\% of relevant studies, with Google Scholar, despite requiring much more time and effort, only yielding another $3 \% .{ }^{57}$ Eighth, while publication bias is possible, all funnel plots performed for every analysis showed no asymmetry. Finally, we did not perform subgroup analyses or metaregression in assessing between-study heterogeneity, as study parameters (such as study design and analytic methods) were often ill-defined and widely variable across this large number of real-world observational studies. ${ }^{58}$

The strengths of this review are the inclusion of all contemporary cohort studies in the troponin era that employed formal definitions of T2MI, analysis of a broader range of variables than those of previous studies, and the more precise discernment of clinically meaningful differences between the two MI populations in patient characteristics, clinical presentation, patterns of care and outcomes. As studies originated from several different jurisdictions, we believe our findings are generalisable to different healthcare systems, although absolute values for some measures did vary between countries. We are aware of a large US cohort study published since completion of our review ${ }^{59}$ which compared patients with T1MI with those with T2MI, but was limited by misclassification bias (relying on administrative hospital discharge data containing an International Classification of Diseases10th Revision code specific for type $2 \mathrm{MI}$, rather than a registry or chart diagnosis based on a formal MI definition), short study period of 3 months in late 2017, and inability to analyse clinical features, investigation results, medication use, coronary anatomy, and postdischarge mortality due to their omission in the datasets.

\section{CONCLUSION}

This review has identified differences between patients with T2MI and T1MI in presenting clinical features, investigation and management profiles and clinical outcomes. These findings may assist clinicians to better recognise T2MI and advise patients about its sequelae, and inform hospital coding and epidemiological trending, quality of care indicators, and interhospital benchmarking of performance relating to the care of patients with T2MI.

The review has also defined persisting gaps in our understanding of the utility and prognostic effects of invasive investigations, revascularisation strategies and cardioprotective medications in patients with T2MI that warrant more randomised trials that enrol such patients.

Contributors All authors contributed to the conception of the work. MK and KW performed the acquisition and analysis of the data. KW and IS were responsible for the interpretation of data. All authors were responsible for drafting manuscript and final approval of the version to be published. All authors agree to be accountable for all aspects of the work in ensuring that questions related to the accuracy or integrity of any part of the work are appropriately investigated and resolved. IS is the guarantor and accepts full responsibility for the work and the conduct of the study, had access to the data, and controlled the decision to publish.

Funding The authors have not declared a specific grant for this research from any funding agency in the public, commercial or not-for-profit sectors.

Competing interests None declared.

Patient consent for publication Not applicable.

Ethics approval This study does not involve human participants.

Provenance and peer review Not commissioned; externally peer reviewed.

Data availability statement All data relevant to the study, except for funnel plots for non-mortality analyses, are included in the article or uploaded as supplementary information. Funnel plots not included are available upon request. 
Supplemental material This content has been supplied by the author(s). It has not been vetted by BMJ Publishing Group Limited (BMJ) and may not have been peer-reviewed. Any opinions or recommendations discussed are solely those of the author(s) and are not endorsed by BMJ. BMJ disclaims all liability and responsibility arising from any reliance placed on the content. Where the content includes any translated material, BMJ does not warrant the accuracy and reliability of the translations (including but not limited to local regulations, clinical guidelines, terminology, drug names and drug dosages), and is not responsible for any error and/or omissions arising from translation and adaptation or otherwise.

Open access This is an open access article distributed in accordance with the Creative Commons Attribution Non Commercial (CC BY-NC 4.0) license, which permits others to distribute, remix, adapt, build upon this work non-commercially, and license their derivative works on different terms, provided the original work is properly cited, appropriate credit is given, any changes made indicated, and the use is non-commercial. See: http://creativecommons.org/licenses/by-nc/4.0/.

\section{ORCID iDs}

Kyle White http://orcid.org/0000-0002-0129-8297

lan Scott http://orcid.org/0000-0002-7596-0837

\section{REFERENCES}

1 Thygesen K, Alpert JS, White HD, et al. Universal definition of myocardial infarction. Circulation 2007;116:2634-53.

2 Thygesen K, Alpert JS, Jaffe AS, et al. Third universal definition of myocardial infarction. Circulation 2012;126:2020-35

3 Thygesen K, Alpert JS, Jaffe AS, et al. Fourth universal definition of myocardial infarction (2018). J Am Coll Cardiol 2018;72:2231-64.

4 Lippi G, Sanchis-Gomar F, Cervellin G. Chest pain, dyspnea and other symptoms in patients with type 1 and 2 myocardial infarction. A literature review. Int J Cardiol 2016;215:20-2.

5 Lippi G, Sanchis-Gomar F, Cervellin G. Cardiac troponins and mortality in type 1 and 2 myocardial infarction. Clin Chem Lab Med 2017;55:181-8.

6 Gupta S, Vaidya SR, Arora S, et al. Type 2 versus type 1 myocardial infarction: a comparison of clinical characteristics and outcomes with a meta-analysis of observational studies. Cardiovasc Diagn Ther 2017;7:348-58.

7 Reid C, Alturki A, Yan A, et al. Meta-Analysis comparing outcomes of type 2 myocardial infarction and type 1 myocardial infarction with a focus on dual antiplatelet therapy. CJC Open 2020;2:118-28.

8 Wang G, Zhao N, Zhong S, et al. A systematic review on the triggers and clinical features of type 2 myocardial infarction. Clin Cardiol 2019;42:1019-27.

9 Moher D, Liberati A, Tetzlaff J, et al. Preferred reporting items for systematic reviews and meta-analyses: the PRISMA statement. PLoS Med 2009;6:e1000097.

10 Riley RD, Higgins JPT, Deeks JJ. Interpretation of random effects meta-analyses. BMJ 2011;342:d549.

11 Stang A. Critical evaluation of the Newcastle-Ottawa scale for the assessment of the quality of nonrandomized studies in metaanalyses. Eur J Epidemiol 2010;25:603-5.

12 Wells BS, D GA, O'Connell JP, Welch V, et al. The Newcastle-Ottawa scale (NOS) for assessing the quality of nonrandomised studies in meta-analyses: Ottawa Hospital research Institute, 2011. Available: http://www.ohri.ca/programs/clinical_epidemiology/oxford.asp

13 Arora S, Strassle PD, Qamar A, et al. Impact of type 2 myocardial infarction (MI) on hospital-level MI outcomes: implications for quality and public reporting. J Am Heart Assoc 2018;7:e008661.

14 Balanescu DV, Donisan T, Deswal A, et al. Acute myocardial infarction in a high-risk cancer population: outcomes following conservative versus invasive management. Int J Cardiol 2020;313:1-8.

15 Baron T, Hambraeus K, Sundström J, et al. Impact on long-term mortality of presence of obstructive coronary artery disease and classification of myocardial infarction. Am J Med 2016;129:398-406.

16 Bonaca MP, Wiviott SD, Braunwald E, et al. American College of Cardiology/American heart Association/European Society of Cardiology/World heart Federation universal definition of myocardial infarction classification system and the risk of cardiovascular death: observations from the TRITON-TIMI 38 trial (trial to assess improvement in therapeutic outcomes by optimizing platelet inhibition with Prasugrel-Thrombolysis in myocardial infarction 38). Circulation 2012;125:577-83.

17 Cediel G, Gonzalez-Del-Hoyo M, Carrasquer A, et al. Outcomes with type 2 myocardial infarction compared with non-ischaemic myocardial injury. Heart 2017;103:616-22.
18 Chapman AR, Adamson PD, Shah ASV, et al. High-Sensitivity cardiac troponin and the universal definition of myocardial infarction. Circulation 2020;141:161-71.

19 Chapman AR, Shah ASV, Lee KK, et al. Long-Term outcomes in patients with type 2 myocardial infarction and myocardial injury. Circulation 2018;137:1236-45.

20 Consuegra-Sánchez L, Martínez-Díaz JJ, de Guadiana-Romualdo LG, et al. No additional value of conventional and high-sensitivity cardiac troponin over clinical scoring systems in the differential diagnosis of type 1 vs. type 2 myocardial infarction. Clin Chem Lab Med 2018;56:857-64.

21 El-Haddad H, Robinson E, Swett K, et al. Prognostic implications of type 2 myocardial infarctions. World J Cardiovasc Dis 2012;02:237-41.

22 Etaher A, Gibbs OJ, Saad YM, et al. Type-li myocardial infarction and chronic myocardial injury rates, invasive management, and 4-year mortality among consecutive patients undergoing high-sensitivity troponin T testing in the emergency department. Eur Heart J Qual Care Clin Outcomes 2020;6:41-8.

23 Furie N, Israel A, Gilad L, et al. Type 2 myocardial infarction in general medical wards: clinical features, treatment, and prognosis in comparison with type 1 myocardial infarction. Medicine 2019;98:e17404.

24 Guimarães PO, Leonardi S, Huang Z, et al. Clinical features and outcomes of patients with type 2 myocardial infarction: insights from the thrombin receptor antagonist for clinical event reduction in acute coronary syndrome (tracer) trial. Am Heart J 2018;196:28-35

25 Hawatmeh A, Thawabi M, Aggarwal R, et al. Implications of misclassification of type 2 myocardial infarction on clinical outcomes. Cardiovasc Revasc Med 2020;21:176-9.

26 Higuchi S, Suzuki M, Horiuchi Y, et al. Higher non-cardiac mortality and lesser impact of early revascularization in patients with type 2 compared to type 1 acute myocardial infarction: results from the Tokyo CCU network registry. Heart Vesse/s 2019;34:1140-7.

27 Javed U, Aftab W, Ambrose JA, et al. Frequency of elevated troponin I and diagnosis of acute myocardial infarction. Am J Cardiol 2009;104:9-13.

28 Kadesjö E, Roos A, Siddiqui A, et al. Acute versus chronic myocardial injury and long-term outcomes. Heart 2019;105:1905-12.

29 Lambrecht S, Sarkisian L, Saaby L, et al. Different causes of death in patients with myocardial infarction type 1, type 2, and myocardial injury. Am J Med 2018;131:548-54.

30 Landes U, Bental T, Orvin K, et al. Type 2 myocardial infarction: a descriptive analysis and comparison with type 1 myocardial infarction. J Cardiol 2016;67:51-6.

31 López-Cuenca A, Gómez-Molina M, Flores-Blanco PJ, et al. Comparison between type-2 and type-1 myocardial infarction: clinical features, treatment strategies and outcomes. J Geriatr Cardiol 2016;13:15-22.

32 Meigher S, Thode HC, Peacock WF, et al. Causes of elevated cardiac troponins in the emergency department and their associated mortality. Acad Emerg Med 2016;23:1267-73.

33 Nestelberger T, Boeddinghaus J, Badertscher P, et al. Effect of definition on incidence and prognosis of type 2 myocardial infarction. J Am Coll Cardiol 2017;70:1558-68.

34 Neumann JT, Sörensen NA, Rübsamen N, et al. Discrimination of patients with type 2 myocardial infarction. Eur Heart $J$ 2017;38:3514-20.

35 Paiva L, Providência R, Barra S, et al. Universal definition of myocardial infarction: clinical insights. Cardiology 2015;131:13-21.

36 Pandey AK, Duong T, Swiatkiewicz I, et al. A comparison of biomarker rise in type 1 and type 2 myocardial infarction. Am J Med 2020;133:1203-8.

37 Putot A, Derrida SB, Zeller M, et al. Short-Term prognosis of myocardial injury, type 1 , and type 2 myocardial infarction in the emergency unit. Am J Med 2018;131:1209-19.

38 Putot A, Jeanmichel M, Chagué F, et al. Type 1 or type 2 myocardial infarction in patients with a history of coronary artery disease: data from the emergency department. J Clin Med 2019;8:2100.

39 Putot A, Jeanmichel M, Chague F, et al. Type 2 myocardial infarction: a geriatric population-based model of pathogenesis. Aging Dis 2020;11:108-17.

40 Radovanovic D, Pilgrim T, Seifert B, et al. Type 2 myocardial infarction: incidence, presentation, treatment and outcome in routine clinical practice. J Cardiovasc Med 2017;18:341-7.

41 Raphael CE, Roger VL, Sandoval Y, et al. Incidence, trends, and outcomes of type 2 myocardial infarction in a community cohort. Circulation 2020;141:454-63.

42 Reed GW, Horr S, Young L, et al. Associations between cardiac troponin, mechanism of myocardial injury, and long-term 
mortality after noncardiac vascular surgery. J Am Heart Assoc 2017;6:e005672.

43 Saaby L, Poulsen TS, Diederichsen ACP, et al. Mortality rate in type 2 myocardial infarction: observations from an unselected Hospital cohort. Am J Med 2014;127:295-302.

44 Saaby L, Poulsen TS, Hosbond S, et al. Classification of myocardial infarction: frequency and features of type 2 myocardial infarction. $A m$ $J$ Med 2013;126:789-97.

45 Sandoval Y, Smith SW, Sexter A, et al. Type 1 and 2 myocardial infarction and myocardial injury: clinical transition to high-sensitivity cardiac troponin I. Am J Med 2017;130:1431-9.

46 Sandoval Y, Thordsen SE, Smith SW, et al. Cardiac troponin changes to distinguish type 1 and type 2 myocardial infarction and 180-day mortality risk. Eur Heart J Acute Cardiovasc Care 2014;3:317-25.

47 Sato R, Sakamoto K, Kaikita K, et al. Long-Term prognosis of patients with myocardial infarction type 1 and type 2 with and without involvement of coronary vasospasm. J Clin Med 2020;9:1686.

48 Shah ASV, McAllister DA, Mills R, et al. Sensitive troponin assay and the classification of myocardial infarction. Am J Med 2015;128:493-501.

49 Singh A, Gupta A, DeFilippis EM, et al. Cardiovascular Mortality After Type 1 and Type 2 Myocardial Infarction in Young Adults. J Am Coll Cardiol 2020;75:1003-13.

50 Smilowitz NR, Subramanyam P, Gianos E, et al. Treatment and outcomes of type 2 myocardial infarction and myocardial injury compared with type 1 myocardial infarction. Coron Artery Dis 2018;29:46-52.
51 Stein GY, Herscovici G, Korenfeld R, et al. Type-II myocardial infarction--patient characteristics, management and outcomes. PLOS One 2014;9:e84285.

52 Truong HH, Victor MV, Imad MA. Mortality and morbidity associated with type 2 myocardial infarction: a single-center study. Annals of Clinical Cardiology 2020;2:70-9.

53 Alpert JS, Thygesen KA, White HD, et al. Diagnostic and therapeutic implications of type 2 myocardial infarction: review and commentary. Am J Med 2014;127:105-8.

54 Gaggin HK, Liu Y, Lyass A, et al. Incident type 2 myocardial infarction in a cohort of patients undergoing coronary or peripheral arterial angiography. Circulation 2017;135:116-27.

55 Lambrakis K, French JK, Scott IA, et al. The appropriateness of coronary investigation in myocardial injury and type 2 myocardial infarction (Act-2): a randomized trial design. Am Heart $J$ 2019;208:11-20.

56 Hartikainen TS, Sörensen NA, Haller PM, et al. Clinical application of the 4th universal definition of myocardial infarction. Eur Heart $J$ 2020;41:2209-16.

57 Bramer WM, Rethlefsen ML, Kleijnen J, et al. Optimal database combinations for literature searches in systematic reviews: a prospective exploratory study. Syst Rev 2017;6:245-56.

58 Metelli S, Chaimani A. Challenges in meta-analyses with observational studies. Evid Based Ment Health 2020;23:83-7.

59 McCarthy CP, Kolte D, Kennedy KF, et al. Patient characteristics and clinical outcomes of type 1 versus type 2 myocardial infarction. J Am Coll Cardiol 2021;77:848-57. 